\title{
Multi-objective Optimal Load Distribution Based on Decomposition-Coordination Method of Large Scale Systems
}

\author{
Liu Kang', Lu Yu ${ }^{2}$, Wang Hai Lu ${ }^{3}$, Jiang Feng ${ }^{1}$ \\ 1. Electric Power Rearch Institute, Gansu Elecric Power Corporation, Lanzhou China, 730050 \\ 2. China Nuckear Control System Eegineering Co,LLTD, Beijing, China, 102206 \\ 3. Economic and Technical Research Institete, Gansu Elecric Power Corporation, Lanzhou China, 730050
}

【Abstract】 This paper divides a model of complicated system which includes numbers of fossil-fueled power plants into a two-tier structure model with coupled subsystems, using decomposition-coordination method of large scale systems. The method consists of Lagrangian relaxation, sub-gradient algorithm and particle swarm optimization (PSO). First, an optimal load distribution model on economy and pollution is proposed. Second, the model is decomposed via Lagrangian relaxation, and PSO is adopted to obtain the optimal solution of each subsystem. Third, the sub-gradient algorithm is used to update the Lagrangian multiplier to coordinate the coupling relation among subsystems. Finally, through iteration of sub-gradient algorithm and PSO, the simulation example realizes the goal that the power plants can obtain the maximum economic efficiency in the case of the minimum pollutant emissions.

【Keywords】 large scale systems; decomposition-coordination; multi-objective; load distribution

\section{Introduction}

In the regional power system of our country, each region has a number of fossil fuel power plants containing multiple generating units. In order to achieve maximum economic interest with minimum environment cost, how to determine the distribution of the load among these units rationally is currently a significant problem faced by the power system dispatching center. The load distribution of all fossil fuel power plants in a region is a high-dimensional, multi-objective and highly coupled problem. It encounters great difficulties in digital computing, such as requiring large memory size, costing a lot of time, even facing "curse of dimensionality" "1] when traditional methods are applied to solve this kind of problems. However, the decomposition-coordination theory of large scale systems is a very effective method to deal with such problems. It can simplify a high-dimensional object, reduce its order, overcome difficulties mentioned above and realize multi-level hierarchical control ${ }^{[2]}$ towards a complex system.

In this paper, a group of fossil fuel power plants are taken into account to establish a multi-objective optimization model of load distribution based on economic interest and environment cost. The Lagrangian relaxation method ${ }^{[3]}$, sub-gradient algorithm $^{[4]}$ and PSO algorithm ${ }^{[5]}$ are combined with each other to form decomposition-coordination method. Firstly, the Lagrangian relaxation method divides the model into a two-tier structure model. Then, sub-gradient algorithm and PSO are adopted to obtain the optimal solution of the upper and lower layers respectively. In the end, through iterations of sub-gradient algorithm and PSO, the optimal distribution result is achieved. The key structure of decomposition-coordination method is shown in Figure 1. 


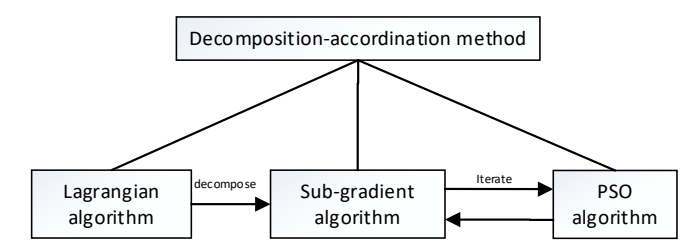

Fig 1 the structure of decomposition-coordination method

At present, domestic power load scheduling is arranged to get maximum economic benefits, ignoring the environmental costs caused by pollutant emissions. In [6], a load distribution model based on speediness and economy in power plants was built and the result was achieved by dynamic programming algorithm, providing a practical and effective basis for load dispatch. The Lagrangian relaxation was adopted to handle unit commitment without considering pollutant emissions in [7], [8], [9]. Among them, satisfactory results was achieved in [7] via aggregative projection sub-gradient method to update Lagrange multiplier, avoiding oscillatory occurrence. Zhang $^{[8]}$ adopted the dichotomy with unit ramp constraint to realize economic dispatch after single unit Pareto optimum had obtained. $\mathrm{Ge}^{[9]}$ adopted differential evolution algorithm to update Lagrangian multiplier and achieved multi-objective global optimal solution. Yuan ${ }^{[10]}$ built a load distribution model which considering coal consumption, pollutant emission and grid revenue as its optimization goals simultaneously. Although [10] gained satisfactory result via using PSO directly, its method would be faced with "curse of dimensionality" in high-dimensional and complex systems.

The decomposition-coordination method proposed in this paper splits the high-dimensional and multi-constrained system into several low-dimensional and single constrained subsystems, not only simplifying the complexity of system but also improving the efficiency of calculation. Eventually, this paper provides a scientific basis for policy-makers to find a most appropriate distribution result to balance the tangle between economy and pollution.

\section{The Model of Load Distribution}

1) The sub-objective functions

2) Constrains

$$
\begin{aligned}
& \text { Coal consumption: } F_{1}=\sum_{j=1}^{N} f_{1}\left(P_{j}\right) \\
& \text { Pollutant emission: } F_{2}=\sum_{j=1}^{N} f_{2}\left(P_{j}\right)
\end{aligned}
$$

$$
\text { Power balance: } \sum_{j=1}^{N} P_{j}(t)=P_{d}
$$

Power limits of each unit: $P_{\operatorname{minj}} \leq P_{j} \leq P_{\max j}$

where $P_{j}(\mathrm{MW})$ stands for the output of unit $\mathrm{j} ; f_{1}\left(P_{j}\right)=a_{j} P_{j}^{2}+b_{j} P_{j}+c_{j}$ (kg) represents coal consumption per unit time of unit $\mathrm{j}$ under the load $P_{j} \cdot a_{j}\left(\mathrm{~kg} /(M W)^{2} \mathrm{~h}\right), b_{j}$ $(\mathrm{kg} / \mathrm{MWh})$ and $c_{j}(\mathrm{~kg})$ are factors of coal consumption of each unit, $f_{2}\left(P_{j}\right)=\xi_{j} P_{j}^{2}+\mu_{j} P_{j}+\gamma_{j}(\mathrm{~kg})$ represents pollutant emission per unit time of unit $\mathrm{j} . \xi_{j}\left(\mathrm{~kg} /(M W)^{2} \mathrm{~h}\right), \mu_{j}(\mathrm{~kg} / \mathrm{MWh}), \gamma_{j}(\mathrm{~kg})$ are factors of pollutant emission of each unit; $\mathrm{N}$ is the total number of units; $P_{d}$ is total load; $P_{\min j}$ is power lower limit of unit $\mathrm{j}$ and $P_{\text {max } j}$ is its power upper limit.

For the reason that coal consumption and pollutant emission have the same units, the data don't need to be treated dimensionally. Hence, the objective function is 
simply a weighted sum of sub-objective functions and the final mutil-objective model is described as follows:

$$
\begin{aligned}
\min & F=\omega_{1} F_{1}+\omega_{2} F_{2} \\
\text { s.t. } & \sum_{j=1}^{N} P_{j}(t)=P_{d} \\
& P_{\min , j} \leq P_{j} \leq P_{\max , j}
\end{aligned}
$$

where $\omega_{1} 、 \omega_{2}$ are weight coefficients ${ }^{[11]}$ between 0 and 1 . Besides, $\omega_{1}+\omega_{2}=1$.

\section{The Decomposition-Coordination Theory of Large Scale Systems}

In the traditional process of solving linear programming or nonlinear programming, the amount of calculations would surge when the dimension of the problem increases, bringing about the curse of dimensionality. The main idea of decomposition-coordination is to decompose a complex system into a bunch of small systems that are low-dimensional and constrained simply. And the coupling relations among these problems can be coordinated separately, not only avoiding the curse of dimensionality but also reducing the difficulty to solve.

\section{A. The Lagrangian Relaxation}

The Lagrangian relaxation algorithm is the key step of decomposition-coordination method to decompose a complex system. It uses the idea of Lagrangian multipliers to relax some complex constraints, dividing the whole system into several subsystems which are separable from each other but can be summed according to different weights ${ }^{[12]}$. Since each subsystem is a low-dimensional mathematical programming problem with simple constraints, the difficulty to obtain the exact solution is greatly reduced.

Based on the multi-objective model as mentioned earlier, Lagrangian multipliers are used to relax the power balance constraint (2) to form Lagrangian function shown as follows:

$$
\begin{aligned}
\ell(P, \beta)= & \omega_{1} \sum_{j=1}^{N}\left(a_{j} P_{j}^{2}+b_{j} P_{j}+c_{j}\right) \\
& +\omega_{2} \sum_{j=1}^{N}\left(\xi_{j} P_{j}^{2}+\mu_{j} P_{j}+\gamma_{j}\right) \\
& +\beta\left(\sum_{j=1}^{N} P_{j}-P_{d}\right)
\end{aligned}
$$

where $\beta$ is the Lagrangian multiplier.

Now, the original problem is equivalent to following equation:

$$
\min F=\min _{P} \max _{\beta} \ell(P, \beta)
$$

By rewrite equation (4) we can get

$$
\begin{aligned}
\ell(P, \beta)= & \sum_{j=1}^{N}\left\{\left(\omega_{1} a_{j}+\omega_{2} \xi_{j}\right) P_{j}^{2}\right. \\
& +\left(\omega_{1} b_{j}+\omega_{2} \mu_{j}+\beta\right) P_{j} \\
& \left.+\omega_{1} c_{j}+\omega_{2} \gamma_{j}\right\}-\beta P_{d}
\end{aligned}
$$

From equation (6), we can see that when the value of $\beta$ is determined, the original problem can be separated to $\mathrm{N}$ subproblems which are independent to each other. According to the theory of Lagrange duality, seeking solutions of equation (5) can be converted into seeking solutions of its dual problem as following equation (7):

$$
\max h(\beta)=\max _{\beta} \min _{P} \ell(P, \beta)
$$

So far, a two hierarchical optimal structure of decomposition and coordination has been built as shown in Figure 2. Its lower layer is n subsystems without any 
coupling relationships. And its upper layer plays a role of a coordinator that receives all feedback variables exported from lower subsystems and outputs a new Lagrangian multiplier called coordinating variable to lower subsystems for the benefit of reaching the overall objective. Then, the subsystems take new multiplier into calculation and output new feedback variables. Finally, an optimal solution can be reached by continually iterating in this way. In essence, decomposition-coordination is an iterative optimization process that exchanges information constantly between the lower and upper layer.

Afterwards, sub-gradient algorithm is used to calculate coordinating variable in the upper layer and PSO is adopted to obtain feedback variables in the lower layer.

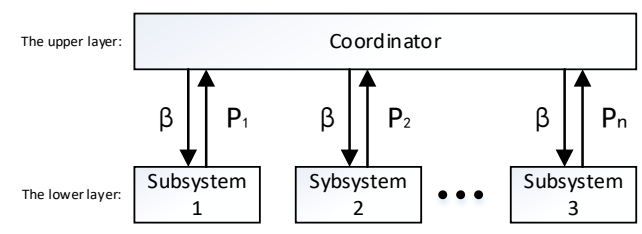

Fig 2 the two-tier structure model with coupled subsystems

In equation (7), $\operatorname{maxh}(\beta)$ is the objective function of the upper coordinator and $\min _{P}(P, \beta)$ is the objective function of the lower subsystems. Obviously, $\beta$ is the coordinating variable and $\mathrm{P}$ are the feedback variables.

Because the value of $\beta$ has been obtained from the coordinator, $\beta P_{d}$ is a fixed value in lower layer. As a result, the objective function for each subsystem can also be written as follows.

$$
\begin{aligned}
& \min \quad \ell_{j}\left(P_{j}\right)=\left(\omega_{1} a_{j}+\omega_{2} \xi_{j}\right) P_{j}^{2} \\
&+\left(\omega_{1} b_{j}+\omega_{2} \mu_{j}+\beta\right) P_{j} \\
&+\omega_{1} c_{j}+\omega_{2} \gamma_{j} \quad(8) \\
& \text { s.t. } \quad P_{\min , j} \leq P_{j} \leq P_{\max , j}
\end{aligned}
$$

\section{B. Update Coordinating Variable}

There is sub-gradient algorithm, Newton algorithm and conjugate gradient algorithm ${ }^{[14]}$ that can be applied to update coordinating variable. The reason why this paper chooses sub-gradient method is that it is straightforward and easy to be embedded in PSO.

As described in [15], Lagrange function $\ell(P, \beta)$ is a linear function of $\beta$. Here, the result of equation (9) is a sub-gradient of $h(\beta)$ for a fixed value of $\beta$.

$$
g(P)=\sum_{j=1}^{N} P_{j}(t)-P_{d}
$$

Based on the equation above, the sub-gradient method is realized by iterating the following equation (10).

$$
\beta^{k+1}=\beta^{k}+s^{k} g^{k}(P)
$$

where $s^{k}$ stands for a positive step size whose value is between 0 and 1 .

In the beginning of iterations, a large step size leads to good convergence, when the value of objective function is far away from its extreme point. However, at the end of iterations, a tiny step size keeps convergence fine. Besides, a proper initial value of $\beta$ is of great benefit to the convergence of the method, contributing to reduce the total computing time.

\section{Update Feedback Variables}

PSO is an intelligent optimization algorithm based on heuristic random search with high operability. The algorithm, whose theory is simple and easy to carry out, initializes a group of random solutions and renews these solutions constantly to search for an optimal choice. In this paper, several particle swarms are initialized with 
different search spaces, which are determined by the upper and lower power limits of units, making all intermediate solutions feasible. The process of PSO is introduced briefly as follows ${ }^{[16]}$.

A swarm contains numbers of particles that all possess position vectors and velocity vectors. These vectors have the same dimension with solutions. Due to position vectors are usually regarded as solutions to the problem, one particle can stand for one solution. In addition, there is a fitness function in each swarm to estimate a particle's performance.

The position vectors and velocity vectors can be described as follows.

$$
\begin{gathered}
X_{i}=\left[x_{i 1}, x_{i 2}, \cdots, x_{i d}\right] \\
V_{i}=\left[v_{i 1}, v_{i 2}, \cdots, v_{i d}\right], i=1,2, \cdots, m
\end{gathered}
$$

where $X_{i}$ and $V_{i}$ are position vectors and velocity vectors of $i(t h)$ particle, $m$ is the number of particles and $\mathrm{d}$ is the number of dimension.

At each iteration, every particle updates its position on the basis of current optimal positions which consist of the globally best position of swarm and the personal best position of particle. The equations to update positions and velocity are written as follows:

$$
\begin{aligned}
v_{i j}(t+1)= & w v_{i j}(t)+c_{1} r_{1}\left[y_{i j}-x_{i j}(t)\right] \\
& +c_{2} r_{2}\left[y_{g j}-x_{i j}(t)\right] \\
x_{i j}(t+1)= & x_{i j}(t)+v_{i j}(t) \\
& j=1,2, \cdots, d
\end{aligned}
$$

where $w$ is inertia factor, $c_{1}$ and $c_{2}$ are nonnegative learning factors, $r_{1}$ and $r_{2}$ are random numbers between 0 and $1, Y_{i}=\left[y_{i 1}, y_{i 2}, \cdots, y_{i d}\right]$ stands for the personal best position of particle i, and $Y_{g}=\left[y_{g 1}, y_{g 2}, \cdots, y_{g d}\right]$ stands for the globally best position.

In the basic PSO algorithm, $c_{1}$ and $c_{2}$ are constant 2, and $w$ is constant 1 . Here, an advanced PSO, whose factors keep variation during iterations, is adopted. The following equations are used in (11).

$$
\begin{gathered}
w=\left\{\begin{array}{c}
\left(1-\frac{\text { iter }}{\text { max iter }}\right)^{2}, \quad \text { iter } \leq 0.7 * \max \text { iter } \\
0.1,
\end{array}\right. \\
c=c_{\text {init }}+\left(c_{\text {final }}-c_{\text {init }}\right) \frac{\text { iter }}{\max \text { iter }}
\end{gathered}
$$

where iter is the current iteration number, max iter is the maximum iteration number, $c_{\text {init }}$ is initial factor, $c_{\text {final }}$ is final factor. Besides, the initial value of $c_{1}$ is 2.5 and the final value is 0.5 , while $c_{2}$ is on the contrary.

The process of PSO is as follows:

Step1. Initialize the position and velocity of each particle;

Step2. Calculate the value of fitness function for each particle, take its initial position as personal best position and then select the globally best position;

Step3. Update the values of $w, c_{1}$ and $c_{2}$ according to (13) and (14);

Step4. Update the velocity and position of each particle according to (11) and (12);

Step5. Calculate the fitness functions again and then update the personal best positions and the globally best position;

Step6. Judge whether the iteration number of PSO satisfies its maximum number and turn to step3 if so, or terminate the PSO program.

In summary, the Lagrangian relaxation algorithm, the sub-gradient algorithm and PSO are the main contents of the decomposition-coordination method used in this paper. Here, the method relaxes the constraint of power balance first to build subsystems associated with single unit, and then determines an initial value of coordinating variable (the Lagrangian multiplier, $\beta$ ) when the iteration number $\mathrm{k}=1$. 
Next, several particle swarms are initialized and feedback variables, each of which is the current power output in each subsystems, are figured out. After that, sub-gradient algorithm is adopted to work out new coordinating variable that will be used to figure out new feedback variables at next iteration. At the end of each iteration, it is necessary to judge whether the current iteration number $\mathrm{k}$ is equal to its maximum number. Finally, an optimal solution is achieved when the variables keep stable.

\section{Case Study}

This section considers a system consisting of 10 thermal power units in a region to realize optimal load distribution through the decomposition-coordination method. Table 1 shows the basic parameters of these units. The total power output of this system is $3740 \mathrm{MW}$, and its lower limit is 1720MW.

\begin{tabular}{|c|c|c|c|c|c|c|c|c|c|c|}
\hline Unit number & 1 & 2 & 3 & 4 & 5 & 6 & 7 & 8 & 9 & 10 \\
\hline $\begin{array}{l}\text { Unit capacity } \\
\text { (MW) }\end{array}$ & 600 & 600 & 600 & 360 & 360 & 360 & 360 & 150 & 150 & 200 \\
\hline $\begin{array}{l}\text { Load limit } \\
\text { (MW) }\end{array}$ & 300 & 300 & 300 & 150 & 150 & 150 & 150 & 60 & 60 & 100 \\
\hline $\begin{array}{l}\text { CC factor a } \\
(\mathrm{kg} /(\mathrm{MW}) 2 \mathrm{~h})\end{array}$ & 0.0028 & 0.0139 & 0.0083 & 0.0162 & 0.0025 & 0.0152 & 0.0611 & 0.0333 & 0.0494 & 0.0208 \\
\hline $\begin{array}{l}\text { CC factor b } \\
\text { (kg/(MW)h) }\end{array}$ & 273.5 & 258.5 & 268.5 & 294.25 & 307.75 & 296.5 & 278.5 & 311.75 & 312 & 303.75 \\
\hline $\begin{array}{l}\text { CC factor c } \\
(\mathrm{kg})\end{array}$ & 13700 & 14500 & 14100 & 6810 & 6737.5 & 6765 & 6350 & 4635.42 & 5040 & 5416.67 \\
\hline $\begin{array}{c}\text { PE factor } \xi \\
(\mathrm{kg} /(\mathrm{MW}) 2 \mathrm{~h})\end{array}$ & 0.002 & 0.0022 & 0.0026 & 0.003 & 0.0045 & 0.004 & 0.0034 & 0.007 & 0.0068 & 0.0055 \\
\hline $\begin{array}{l}\text { PE factor } \mu \\
\text { (kg/(MW)h) }\end{array}$ & -0.52 & -0.52 & -0.52 & -0.45 & -0.574 & -0.483 & -0.53 & -0.3 & -0.34 & -0.3 \\
\hline $\begin{array}{c}\text { PE factor } \gamma \\
(\mathrm{kg})\end{array}$ & 29.4 & 30.5 & 29.5 & 31.5 & 28.4 & 28.5 & 28.8 & 29.5 & 38.8 & 33.8 \\
\hline
\end{tabular}

In the calculation of this section, the number of particles in each swarm is set to 50, and the maximize iteration number of PSO is set to 50, and the maximize iteration number of decomposition-coordination method is also set to 50. Figure 3 shows the unit load allocation optimization process under a total load of $3000 \mathrm{MW}$ when coal consumption coefficient and pollutant emission coefficient are both 0.5. The horizontal axis stands for the iteration number and the vertical axis represents power output. It can be seen that the method is fully convergent when the iteration number is 15. And the simultaneous change of Lagrangian multiplier is shown in Figure 4. As the same trend as shown in Figure 3, the Lagrangian multiplier keeps unchanged after the 15th iteration. It testifies that the method has fast convergence and high accuracy.

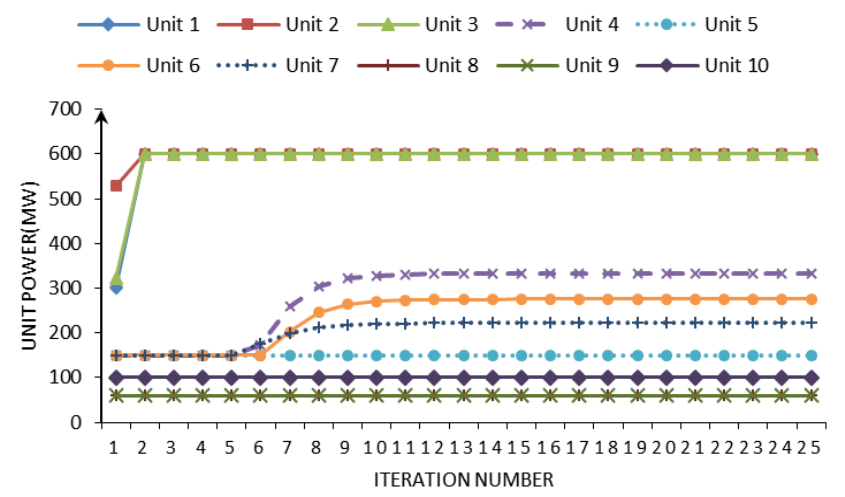

Fig 3 the unit load allocation optimization process under $3000 \mathrm{MW}$ 


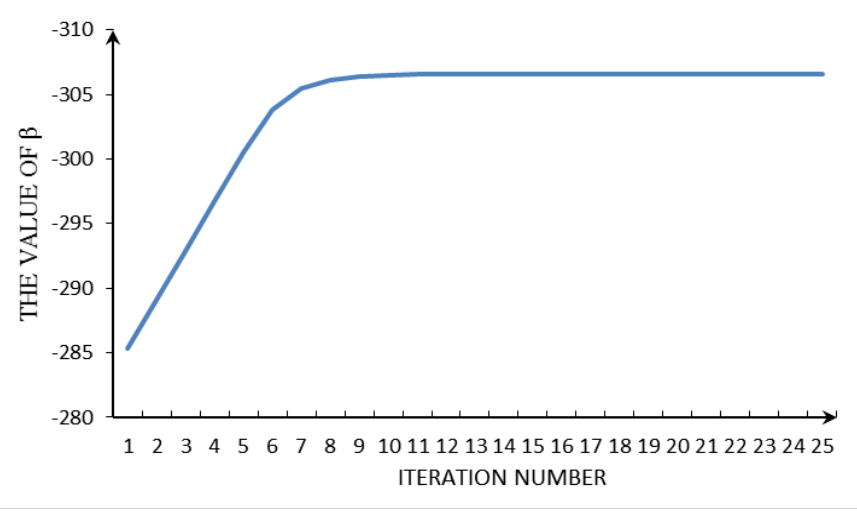

Fig 4 the change of lagrangian multiplier under 3000MW

With a deteriorating ecological environment, it is urgent to reduce environmental cost during industrial production. In the model adopted in this paper, coal consumption weight coefficient and pollutant emission weight coefficient determine the preference of objective function. It is obvious that different coefficients result in different load distribution outcomes. The results of calculation under different coefficients are listed in Table 2 when the total load is 3000MW. And Table 3 shows corresponding coal consumptions and pollutant emissions. From Table 3, we can see that when only coal consumption is preferred $\left(\omega_{1}=1 、 \omega_{2}=0\right)$ in objective function, coal consumption is minimized while pollutant emission is maximized. And when only pollutant emission is preferred ( $\left.\omega_{1}=0 、 \omega_{2}=1\right)$, the result is on the contrary. In addition, when the two coefficients are both 0.5 , the total amount of coal consumption and pollutant emission is minimized.

It turns out that the coal consumption is in conflict with pollutant emission under the same load. That is to say, economic costs and environmental costs are deviate from each other.

Table 4 lists a series of load optimization results under different total loads when the two coefficients are both 0.5 .

Table2 the optimal solution with different weight coefficients under

\begin{tabular}{cc|cccccccccc}
\hline$\omega_{1}$ & $\omega_{2}$ & 机组 1 & 机组 2 & 机组 3 & 机组 4 & 机组 5. & 机组 6 & 机组 7 & 机组 8 & 机组 9 & 机组 10 \\
\hline 0.5 & 0.5 & 600.00 & 600.00 & 600.00 & 332.95 & 150.00 & 275.22 & 221.83 & 60.00 & 60.00 & 100.00 \\
0.8 & 0.2 & 600.00 & 600.00 & 600.00 & 332.73 & 150.00 & 278.95 & 218.32 & 60.00 & 60.00 & 100.00 \\
0.2 & 0.8 & 600.00 & 600.00 & 600.00 & 331.40 & 150.00 & 265.59 & 232.67 & 60.00 & 60.00 & 100.35 \\
1 & 0 & 600.00 & 600.00 & 600.00 & 332.54 & 150.00 & 280.40 & 217.06 & 60.00 & 60.00 & 100.00 \\
0 & 1 & 525.29 & 477.53 & 404.07 & 338.52 & 239.46 & 258.02 & 310.46 & 134.37 & 141.26 & 171.01 \\
\hline
\end{tabular}

Table 3 the coal consumption and emissions with different weight

\begin{tabular}{cccccc} 
& \multicolumn{5}{c}{ coefficients under $3000 \mathrm{MW}$} \\
\hline & $\omega 1=0.5$, & $\omega 1=0.8$, & $\omega 1=0.2$, & $\omega 1=1$, & $\omega 1=0$, \\
$\omega 2=0.5$, & $\omega 2=0.2$, & $\omega 2=0.8$, & $\omega 2=0$, & $\omega 2=1$, \\
\hline Coal consumption & 935185.8 & 935184.1 & 935203.3 & 935184 & 950229.1 \\
Pollutant emission & 2274.664 & 2277.396 & 2267.363 & 2278.451 & 1955.335 \\
Total & 937460.4 & 937461.5 & 937470.6 & 937462.5 & 952184.4 \\
\hline
\end{tabular}


Table 4 the distribution results under different total loads

\begin{tabular}{c|cccccccccc}
\hline Total load & Unit 1 & Unit 2 & Unit 3 & Unit 4 & Unit 5 & Unit 6 & Unit 7 & Unit 8 & Unit 9 & Unit 10 \\
\hline 1720.00 & 300.00 & 300.00 & 300.00 & 150.00 & 150.00 & 150.00 & 150.00 & 60.00 & 60.00 & 100.00 \\
1800.00 & 300.00 & 380.00 & 300.00 & 150.00 & 150.00 & 150.00 & 150.00 & 60.00 & 60.00 & 100.00 \\
1900.00 & 300.00 & 480.00 & 300.00 & 150.00 & 150.00 & 150.00 & 150.00 & 60.00 & 60.00 & 100.00 \\
2000.00 & 300.00 & 540.44 & 339.56 & 150.00 & 150.00 & 150.00 & 150.00 & 60.00 & 60.00 & 100.00 \\
2100.00 & 336.38 & 566.13 & 377.49 & 150.00 & 150.00 & 150.00 & 150.00 & 60.00 & 60.00 & 100.00 \\
2200.00 & 393.90 & 583.28 & 402.82 & 150.00 & 150.00 & 150.00 & 150.00 & 60.00 & 60.00 & 100.00 \\
2300.00 & 451.72 & 600.00 & 428.28 & 150.00 & 150.00 & 150.00 & 150.00 & 60.00 & 60.00 & 100.00 \\
2400.00 & 521.15 & 600.00 & 458.85 & 150.00 & 150.00 & 150.00 & 150.00 & 60.00 & 60.00 & 100.00 \\
2500.00 & 590.57 & 600.00 & 489.43 & 150.00 & 150.00 & 150.00 & 150.00 & 60.00 & 60.00 & 100.00 \\
2600.00 & 600.00 & 600.00 & 580.00 & 150.00 & 150.00 & 150.00 & 150.00 & 60.00 & 60.00 & 100.00 \\
2700.00 & 600.00 & 600.00 & 600.00 & 198.27 & 150.00 & 150.00 & 181.73 & 60.00 & 60.00 & 100.00 \\
2800.00 & 600.00 & 600.00 & 600.00 & 245.91 & 150.00 & 188.18 & 195.91 & 60.00 & 60.00 & 100.00 \\
2900.00 & 600.00 & 600.00 & 600.00 & 289.43 & 150.00 & 231.70 & 208.87 & 60.00 & 60.00 & 100.00 \\
3000.00 & 600.00 & 600.00 & 600.00 & 332.95 & 150.00 & 275.22 & 221.83 & 60.00 & 60.00 & 100.00 \\
\hline 3100.10 & 600.00 & 600.00 & 600.00 & 360.00 & 150.00 & 331.17 & 238.48 & 60.00 & 60.00 & 100.45 \\
3200.00 & 600.00 & 600.00 & 600.00 & 360.00 & 191.14 & 360.00 & 247.15 & 60.00 & 60.00 & 121.71 \\
3300.00 & 600.00 & 600.00 & 600.00 & 360.00 & 263.89 & 360.00 & 255.04 & 60.00 & 60.00 & 141.07 \\
3400.00 & 600.00 & 600.00 & 600.00 & 360.00 & 336.63 & 360.00 & 262.94 & 60.00 & 60.00 & 160.43 \\
\hline 3500.00 & 600.00 & 600.00 & 600.00 & 360.00 & 360.00 & 360.00 & 298.23 & 61.93 & 60.00 & 200.00 \\
3600.00 & 600.00 & 600.00 & 600.00 & 360.00 & 360.00 & 360.00 & 329.52 & 112.02 & 78.46 & 200.00 \\
\hline 3700.00 & 600.00 & 600.00 & 600.00 & 360.00 & 360.00 & 360.00 & 358.29 & 150.00 & 111.47 & 200.00 \\
3740.00 & 600.00 & 600.00 & 600.00 & 360.00 & 360.00 & 360.00 & 360.00 & 150.00 & 150.00 & 200.00 \\
\hline
\end{tabular}

\section{Conclusion}

The decomposition-coordination method presented in this paper, which takes economic factor and environment factor into consideration, performs quite well in load distribution among fossil fuel power plants. The Lagrangian relaxation decomposes the model into several subsystems, avoiding the "curse of dimensionality" and reducing the difficulty of problem. The combination of sub-gradient and PSO not only guarantee all intermediate solutions are feasible to each subsystem, but also ensure that the method has a fast convergence speed. The case study testifies the correctness of the model and the effectiveness of the method, which makes this paper as a new theoretical basis for multi-objective optimal load distribution.

\section{References:}

[1]. Da Qingli.Theory And Methods On Large Scale Systems.Southeast University Press,1989.5

[2]. Tu Xuyan,Wang Cong,Guo Yanhui. Large Systems Cybernetics. Beijing University Of Posts And Telecommunications Press,2005.8

[3]. Marshall L.Fisher. The Lagrangian Relaxation Method For Solving Integer Programming Problems. Management Science,1981(1)

[4]. Tao Jiping,Xu Wenyan,Wang Hao. Incremental Subgradient Method To Scheduling Algorithm Based On Lagrangian Relaxation.Control Engineering Of China,2007(5):566-568

[5]. Qian Feng. Particle Swarm Optimization And Its Industrial Application. Science Press,2013.3

[6]. Wang Zhiguo,Liu Jizhen, Tan Wen,Yang Guangjun. Multi-Objective Optimal Load Distribution Based On Speediness And Economy In Power Plants.Proceedings Of The Csee,2006(19):86-92

[7]. Miao Qing,Cao Guangyi,Zhu Xinjian. A New Algorithm For Unit Commitment Based On Aggregative Projection Subgradient Method.Computer Simulation, 2008(2):245-247

[8]. Zhang Ningyu,Gao Shan,Zhao Xin. A Fast Lagrangian Relaxation Method For Unit Commitment. Power System Protection And Control,2012(19):47-53

[9]. Ge Xiaolin,Zhang Lizi,Wang Nan. Two-Stage Optimization Algorithm For Unit Commitment Problem.Electric Power,2010(4):14-18

[10]. Yuan Ying.Study On The Application Of Particle Swarm Optimization In The System Power Economic Dispatch. Xi'an University Of Technology,2010

[11]. Deng Xue,Li Jiaming,Zeng Haojian,Chen Junyang,Zhao Junfeng. Application of Analytic Hierarchy Weight Calculation Method. Journal of Mathematics in Practice and Theory,2012(7):93-100

[12]. He Xiaoyu,Zhang Lizi,Xie Guohui. Unit Commitment Using An Improved Lagrangian Relaxation Method. Power System Protection And Control,2010(17):16-21

[13]. S.J.Wang,S.M.Shahidehpour,D.S.Kirschen,S.Mokhtari,G.D.Irisarri.Short-Term Generation Scheduling With Transmission And Environmental Constraints Using An Augmented Lagrangian Relaxation. Ieee Transactions On Power Systems,1995(3)

[14]. Xing Zhao, Peter B. Luh, Jihua Wang .The Surrogate Gradient Algorithm For Lagrangian Relaxation Method. Proceedings Of The 36th Conference On Decision \& Control,1997

[15]. Dimitri P.Bertsekas.Network Optimization:Continuous And Discrete Models. Tsinghua University Press,2013.1

[16]. Kai Sedlaczek,Peter Eberhard. Using Augmented Lagrangian Particle Swarm Optimization For Constrained Problems In Engineering. Struct Multidisc Optim 2006(32): 277-286

[17]. Jin S.Heo, Kwang Y.Lee, Raul Garduno-Ramirez. Multiobjective Control Of Power Plants Using Particle Swarm Optimization Techniques. leee Transactions On Energy Conversion,2006(2)

[18]. Qin Zhen. The Multi-Objective Optimization Scheduling Of The System With Multi-Power Sources. Guangxi University,2012 